\title{
Große Erwartungen:
}

\section{Die S3-Leitlinie zur Therapie der Autismus-Spektrum-Störungen}

\author{
Inge Kamp-Becker ${ }^{1}$, Sanna Stroth ${ }^{1}$ und Luise Poustka²
}

\author{
Klinik für Kinder- und Jugend psychiatrie, Psychosomatik und Psychotherapie, Fachbereich Medizin der Philipps-Universität Marburg \\ 2 Klinik für Kinder- und Jugendpsychiatrie und -psychotherapie, Universitätsmedizin Göttingen
}

Laut der Arbeitsgemeinschaft der Wissenschaftlichen Medizinischen Fachgesellschaften e.V. $\left(\mathrm{AWMF}^{1}\right)$ ist die S3-Leitlinie zur Therapie der Autismus-Spektrum-Störungen im Kindes-, Jugend- und Erwachsenenalter im Februar 2021 zu erwarten. Die darin enthaltenen Empfehlungen sind aus dem Evidenzgrad der aktuell vorhandenen Studienergebnisse hervorgegangen. Sie sind in einem langen, gemeinsamen Prozess von einer repräsentativen Kommission relevanter Berufsgruppen und betroffener Menschen (siehe unten) erarbeitet und konsentiert worden. Die Empfehlungen wurden schon vor deren Veröffentlichung sehr unterschiedlich interpretiert, kontrovers diskutiert oder sogar abgelehnt. Der Bundesverband ,autismus Deutschland e.V.“, der Deutsche Fachverband für Verhaltenstherapie e.V. (DVT) und die Deutsche Musiktherapeutische Gesellschaft e.V. haben bereits Sondervoten eingelegt.

Im Folgenden möchten wir einige Überlegungen zur Debatte um die Leitlinie vorlegen und diese mit offenen Fragen zur Diskussion stellen. Leitlinien sind „systematisch entwickelte Aussagen, die den gegenwärtigen Erkenntnisstand wiedergeben, um die Entscheidungsfindung von Ärzten und Patienten für eine angemessene Versorgung bei spezifischen Gesundheitsproblemen zu unterstützen". Ihr vorrangiges Ziel ist die Verbesserung der medizinischen Versorgung durch die Vermittlung von aktuellem Wissen $\left(\mathrm{AWMF}^{2}\right)$.

Die Entwicklung von S3-Leitlinien ist ein aufwendiger, ressourcenfordernder Prozess, der eine ,gewisse Leidensfähigkeit" der an der Entwicklung beteiligten Fachvertreterinnen und -vertreter voraussetzt (Schepker,
2018). Insbesondere die Entwicklung einer evidenz- und konsensbasierten S3-Leitlinie setzt eine klare, systematische, methodische, dokumentierte Vorgehensweise voraus. Wesentliche Elemente sind dabei die Evidenzbasierung (Recherche, Auswahl und Bewertung der Literatur zu klinischen relevanten Fragestellungen sowie eine Einschätzung der Qualität der Studien) und die Konsensbasierung (Diskussion und Abstimmung jeder Empfehlung durch die für den Adressatenkreis repräsentativ zusammengesetzte Leitlinienkommission).

Derzeit ist das Angebot an Autismus-Therapien unterschiedlichster Couleur extrem groß und weit gefächert. Es reicht von gut untersuchten, manualisierten Frühförderprogrammen (Freitag et al., 2020; Poustka \& KampBecker, 2017; Poustka, Poustka \& Kamp-Becker, 2019; Teufel, Wilker, Valerian \& Freitag, 2017) über (in ihrer Evidenz nicht untersuchten aber häufig angewandten) eklektische Verfahren bis zu fragwürdigen „biomedizinischen", gesundheitsschädlichen und ethisch bedenklichen Verfahren. Es gibt Therapieangebote, die bei betroffenen Familien zu einer enormen Entlastung und bei der oder dem Einzelnen zu erheblichen Entwicklungsfortschritten führen können. In einigen Ansätzen sind auch wirksame pharmakologische Interventionen enthalten. Trotz begrenzter Evidenz und in einigen Fällen potenziell schädlicher Wirkung werden außerdem auch komplementär- und alternativmedizinische Ansätze angewandt (Höfer et al., 2019; Höfer, Hoffmann \& Bachmann, 2016). Vor dem Hintergrund dieser Vielfalt an Behandlungsmöglichkeiten besteht zweifelsohne ein Bedarf an klaren Entscheidungshilfen mit konkreten, evidenzba- 
sierten Handlungsempfehlungen, um die Versorgungssituation zu verbessern. Sowohl betroffene Familien als auch in der Versorgung Tätige benötigen solide, wissenschaftlich belegte Informationen, um zu informierten Entscheidungen zu kommen. Die gegenwärtige Darstellung von Autismus in den Medien ist diesbezüglich unzureichend und weist deutliche Qualitätsmängel auf (Kamp-Becker, Stroth, Stehr \& Weber, 2020).

- Impliziert die Entwicklung einer Leitlinie die Annahme, dass das zu „Verbessernde“ (= gegenwärtige Versorgungssituation) grundsätzlich schlecht ist? Resultiert die ablehnende Haltung gegenüber der S3-Leitlinie aus der ihr implizit zugeschriebenen mangelnden Wertschätzung der gegenwärtigen Versorgungssituation?

Zur Therapie von Autismus-Spektrum-Störungen liegt eine überwältigende Zahl von Studien vor. Die Forschungslage ist also deutlich besser als bei manchen anderen Störungsbildern (z.B. Störung des Sozialverhaltens, Angststörungen). Im Rahmen der Leitlinienentwicklung fließen jedoch in die Entscheidungen zu Handlungsempfehlungen neben empirischen Befunden auch Erfahrungen, subjektive Einschätzungen und Wertvorstellungen der Leitlinienkommission ein. Dies ist insbesondere bei der klinischen Beurteilung der Aussagefähigkeit und Anwendbarkeit der Evidenz der Fall oder wenn nicht genügend bzw. keine Studien zu einer bestimmten Fragestellung vorliegen (Kopp, Selbmann \& Koller, 2007). Hieraus ergeben sich möglicherweise ebenfalls Akzeptanzprobleme für die Leitlinie. Wenn für Therapiemethoden oder -ansätze noch keine oder in ihrer Qualität unzureichende Studien vorliegen, um die Evidenz hinreichend beurteilen zu können, kann die Leitlinie keine entsprechende evidenzbasierte Empfehlung formulieren. Aus Sicht der in der Versorgung Tätigen belegt jedoch ihre subjektive Erfahrung die Wirksamkeit der von ihnen angewandten Interventionen, ohne dass hierzu empirische Belege vorliegen. Natürlich ist „keine Evidenz “ nicht gleichbedeutend mit Unwirksamkeit. Dennoch scheint dies aus Sicht der in der Versorgung Tätigen ein relevanter und empfindsamer Punkt zu sein, der zu geringerer Akzeptanz der Leitinie beitragen könnte. Dass die subjektive Realität bzw. das „Erfahrungswissen" nicht immer mit empirischen Befunden übereinstimmt, sehen wir auch in anderen Bereichen: Ärztinnen und Ärzte verschreiben z.B. besonders gerne bestimmte Antidepressiva, die sie aus Erfahrung gut ken- nen, auch wenn die Evidenz andere Antidepressiva als besser einstuft (Bauer et al., 2008).

- Ist der bestehende Dissens in der Einschätzung der Leitlinie auf die Nichtübereinstimmung zwischen „Erfahrungswissen“ und „empirisch belegtem Wissen“ zurückzuführen?

Dies bringt eine Diskussion um den Begriff der „Wirksamkeit" auf. Als Wirksamkeit wird allgemein das Ausmaß bezeichnet, in dem etwas (die gewünschte) Wirkung zeigt und den erhofften Nutzen hat. In der Medizin wird Wirksamkeit als die Fähigkeit eines Verfahrens oder einer Substanz bezeichnet, kausal eine - meist vorab definierte Veränderung hervorzurufen. Im Bereich der Interventionsforschung wird Wirksamkeit als ursächlich auf eine Intervention rückführbare Differenz eines Zustands im Vergleich $\mathrm{zu}$ einem unbeeinflussten oder anders beeinflussten Zustand verstanden ${ }^{3}$. Eine derartige Kausalität zwischen Intervention und Zielerreichung lässt sich durch randomisierte, kontrollierte Studien belegen, bei denen man sich bemüht, diesen Zusammenhang möglichst objektiv, reliabel und valide darzustellen. Im Alltag hängt die Wirksamkeit einer Maßnahme/Intervention bei einer konkreten Person jedoch von einer Vielzahl von Faktoren $\mathrm{ab}$ (individuelle Motive, Vorstellungen, Wünsche, soziales Umfeld, Ressourcen usw.). Die Wirkung ist oft schwer zu objektivieren bzw. wird nicht standardisiert erfasst. Somit wird eine Wirkung nicht zwingend als kausaler Zusammenhang begriffen bzw. bestehen Zweifel, ob sich die Aussagen aus empirischen Studien eindeutig auf eine bestimmte Person übertragen lassen.

- Entsteht aus dem unterschiedlichen Verständnis von Wirksamkeit ein unüberbrückbarer Graben zwischen der Realität subjektiver Erfahrung und empirischer Forschung?

Um die Entscheidungen der Leitlinienkommission gegen eine Vielzahl unerwünschter (Verzerrungs-)Effekte abzusichern, wird der Entwicklungsprozess von einer geschulten AWMF-Leitlinienberaterin (Frau Kopp ${ }^{4}$ ) begleitet. Im Sinne einer redaktionellen Unabhängigkeit müssen alle stimmberechtigen Mitglieder der Leitlinienkommission potenzielle Interessenkonflikte offenlegen. Als solche werden Gegebenheiten definiert, die das professionelle Urteilsvermögen oder Handeln durch ein sekundäres Interesse unangemessen beeinflussen können ${ }^{5}$. Sekundäre In-

\footnotetext{
https://www.dvfr.de/fileadmin/user_upload/DVfR/Downloads/Stellungnahmen/DVfR_Stellungnahme_BTHG_Wirkung_u_Wirksamkeit_bf.pdf (abgerufen am 09.12.2020).

4 An dieser Stelle vielen Dank an Frau Kopp für ihre unermüdliche Geduld mit der Kommission und deren stundenlangen Diskussionen, die sie moderiert und strukturiert hat.

5 https://www.awmf.org/leitlinien/awmf-regelwerk/ll-entwicklung/awmf-regelwerk-01-planung-und-organisation/po-interessenkonflikte/interessenskonflikte.html (abgerufen am 09.12.2020).
} 
teressen sind z.B. direkte und indirekte finanzielle, akademische, klinische, persönliche Gegebenheiten. Dabei geht es neben Interessenkonflikten, die sich durch die Zusammenarbeit z.B. mit Arzneimittelkonzernen ergeben, aber auch um ideelle (z.B. Krankheitsverständnis, Überzeugungen), wirtschaftliche (z.B. Tätigkeit in einer Institution, die Therapie anbietet; Autorschaft von Therapiemanualen), akademische (z.B. Ausbildungs-/Supervisionstätigkeit), klinische (z. B. Ausbildung in einer bestimmten Therapieform) oder persönliche Interessen (z. B. Auswirkungen der Empfehlungen auf die eigene Lebenslage).

- Ist es nicht so, dass die Evidenzbasierung die Empfehlungen aufwertet, da die wissenschaftlich Studienlage und nicht die Interessenlage von Einzelnen oder Gruppen im Vordergrund steht?

- Sind die in der Leitlinie dargestellten Empfehlungen insbesondere wenn keine ausreichenden Studien vorliegen - ein Spiegel eines gefundenen Konsens und somit das Meinungsbild der Mehrheit, die sich durchsetzen konnte?

Ein weiterer wichtiger Punkt bei der Entwicklung von Leitlinien ist die Repräsentativität der Leitlinienkommission mit Fachleuten aus allen für das jeweilige Störungsbild wichtigen Berufsgruppen einschließlich Vertreterinnen und Vertretern von Betroffenen. Die Zusammenstellung der Berufsgruppen impliziert jedoch, dass auch die mit eingeschlossen werden, deren Arbeit von den Aussagen der Leitlinie betroffen sein könnte. Wird in der Leitlinie beispielsweise eine Therapieform nicht empfohlen oder als nicht wirksam eingeschätzt, so könnte dies unmittelbaren Einfluss auf ihre Arbeit und somit auf ihre berufliche Existenz nehmen, d.h. sekundäre Interessen betreffen.

Der Einbezug von Vertreterinnen und Vertretern von Betroffenen ist gerade beim breiten Störungsspektrum des Autismus keine triviale Angelegenheit. Bei der Mehrheit der Betroffenen finden wir eine unterdurchschnittliche Intelligenz und bei ca. einem Drittel eine mehr oder weniger stark ausgeprägte Intelligenzminderung. Von diesen Menschen verfügen viele über kein oder ein sehr eingeschränktes Sprachvermögen (Arbeitsgemeinschaft der Wissenschaftlichen Medizinischen Fachgesellschaften [AWMF] - Ständige Kommission Leitlinien, 2016), sodass sie ihre Perspektive nur unzureichend mit in die Leitlinienentwicklung einbringen können. Daher waren bei der S3-Leitlinie zur Therapie der Autismus-SpektrumStörungen in der Kommission nur „Aspies“e.V. vertreten, bei denen es sich i.d.R. um Erwachsene handelt, die sich selbst sehr stark mit dem Störungsbild identifizieren ${ }^{6}$ und sich sehr deutlich für eine Berücksichtigung ihrer Bedürf- nisse in der Gesellschaft und im Gesundheitssystem einsetzen. Der Bundeverband „autismus Deutschland e.V.“ war ebenfalls vertreten, jedoch in Form von Leiterinnen und Leitern von Autismus-Therapiezentren und nicht von Elternvertreterinnen und -vertretern. Die überwiegende Mehrheit der Betroffenen und ihrer Angehörigen war also in der Leitlinienkommission nicht vertreten.

- Wird die Arbeit der Leitlinienkommission schon durch die Auswahl der Mitglieder beeinflusst? Welche Bedeutung hat die Konstellation der Teilnehmenden der Konsensuskonferenz auf die Empfehlungen? Wird der Moderationsprozess dadurch beeinflusst?

Die Implementierung einer neuen Leitlinie ist keine leichte Aufgabe. Es bedarf einer klaren, behutsamen und wertschätzenden Kommunikation, um Handlungsempfehlungen in individuelles Handeln in der Versorgung zu übertragen und möglicherweise eine Veränderung etablierter Vorgehensweisen zu erzielen. Ohne Zweifel ist die professionelle Akzeptanz einer Leitlinie erhöht, je besser die methodischen, fachlichen und inhaltlichen Anforderungen bei der Leitlinienerstellung erfüllt wurden. Letzteres ist bei den S3-Leitlinien zur Therapie der AutismusSpektrum-Störungen der Fall. Das übergeordnete Ziel der Leitlinien, die Verbesserung der Versorgung durch die Vermittlung von aktuellem Wissen, hängt jedoch auch stark von der Akzeptanz der in der Versorgung Tätigen ab, ob sie die systematisch entwickelten Aussagen, die den gegenwärtigen Kenntnisstand wiedergeben, nicht als Bedrohung ihrer langjährigen klinischen Praxis erleben, sondern als Chance für eine Verbesserung der Versorgung.

Hierzu sind u.E. zwei Aspekte relevant: Einerseits die Grenzen der Leitlinie in ihrer Anwendbarkeit und zweitens die Struktur der S3-Leitlinie. Grundsätzlich ist es so, dass das es nicht das Ziel von Leitlinien ist, mit ihren Empfehlungen die aktuelle klinische Praxis dauerhaft fortzuschreiben und sozialrechtliche Bedingungen für die Abrechnung bestimmter Leistungen zu schaffen (Schepker, 2018). Leitlinien sollen als Entscheidungshilfe mit konkreten Handlungsempfehlungen dienen, die auf der Basis von aktuellem und in seiner Qualität beurteiltem Wissen beruhen. Das Ziel ist es, zur Verbesserung der Versorgung beizutragen und den Betroffenen eine möglichst evidenzbasierte Behandlung zu ermöglichen. Leitlinien geben lediglich „Handlungs- und Entscheidungskorridore“ vor, von denen in begründeten Fällen abgewichen werden kann oder sogar muss. Die Anwendbarkeit muss in der individuellen Situation geprüft werden nach dem Prinzip der Indikationsstellung, Beratung, Präferenzermittlung usw. ${ }^{3}$.

6. https://www.aspies.de/ (abgerufen am 09.12.2020). 
Wenn die Empfehlungen der Leitlinie für ein Individuum als nicht geeignet angesehen werden, sollte dies begründet und eine andere Empfehlung ausgesprochen werden. Der Grundsatz der Beurteilung des Einzelfalls gilt insbesondere auch für Unterstützung, die über die Eingliederungshilfe finanziert wird. Denn für die Frage, ob Eingliederungshilfe gewährt wird oder nicht, ist entscheidend ob die Förderung im konkreten Einzelfall geeignet erscheint, die Folgen einer Behinderung zu beseitigen oder zu mildern. Das bedeutet, dass der Hilfebedarf im Einzelfall geklärt werden muss. Leitlinien hingegen können nicht auf jedwede individuellen Umstände eingehen. Eine Leitlinie ist rechtlich nicht bindend und hat daher weder haftungsbegründende noch haftungsbefreiende Wirkung (Hübner \& Wienke, 2019).

Im Recht der Eingliederungshilfe, das sich ab dem Jahr 2020 komplett in Teil II des SGB (Sozialgesetzbuch) IX befindet, wird gefordert, dass die Leistungserbringer ein Qualitätsmanagement sicherstellen müssen, welches durch zielgerichtete und systematische Verfahren und Maßnahmen die Qualität der Versorgung gewährleistet und kontinuierlich verbessert ( $\$ 37$ Abs. 2 S. 1 SGB IX). Dabei sind nach $₫ 38$ SGB IX einheitliche Grundsätze der Wirksamkeit, Zweckmäßigkeit und Wirtschaftlichkeit zu berücksichtigen. Der Wirkungsbegriff wird dabei so verstanden, dass neben „der Erreichung der allgemeinen, in $\$ 4$ Abs. 1 Nr. 1-4 SGB IX genannten Ziele der Leistungen zur Teilhabe“ die „Wirkung anhand der Überprüfung von individuellen Teilhabezielen [ $\$ \mathbb{S} 13$ Abs. 2 Nr. 3 SGB IX, 19 Abs. 3 S. 1 SGB IX, 144 Abs. 2 S. 1 SGB XII] dargestellt werden kann. Eine positive Wirkung von im Rahmen der Eingliederungshilfe erbrachten Leistungen kann dementsprechend angenommen werden, wenn individuelle, also auf die konkrete, leistungsberechtigte Person bezogene Teilhabeziele erreicht werden"7.

Gerade den Aspekt der Zieldefinition und -überprüfung greift die S3-Therapieleitlinie auf, indem sie ein hohes Maß an Differenziertheit und Strukturierung in den Empfehlungen aufweist, welches als herausragend bezeichnet werden kann. Empfohlen wird ein klares und definiertes Vorgehen in der Versorgung:

1. Fallmanagement: Interventionen/Maßnahmen sollten von einer Hand koordiniert, vernetzt und abgestimmt werden, um Lücken, Dopplungen, unwirksame oder sogar schädliche Interventionen zu vermeiden. Es sollte durch approbierte Fachleute nach einer qualifizierten Diagnostik entsprechend der Leitlinie ein langfristiges „Fallmanagement" und indizierte Verlaufsuntersuchungen durchführt werden mit dem Ziel, im
Einzelfall bedarfsentsprechend die nötige Unterstützung, Behandlung, Begleitung und/oder Förderung zugänglich zu machen.

2. Festlegung der Therapieziele: Jede Therapie und Intervention soll mit klar definierten, konkreten, in der Regel hierarchisch formulierten Therapiezielen sowie für einen befristeten Zeitraum geplant werden, d.h., es geht bei der Therapieplanung um das sukzessive Erreichen kurz- und langfristiger, konkreter Therapieziele, die überprüft und - je nach Notwendigkeit - auch angepasst werden müssen (s.u.).

3. Auswahl der Intervention: Menschen mit AutismusSpektrum-Störung und ihren Familien sollen bei entsprechender Indikation die in dieser Leitlinie empfohlenen Interventionen angeboten werden. Bei der Auswahl der Intervention ist entscheidend, um welche Zielsymptome es sich zum Zeitpunkt der konkreten Therapieplanung handelt. Die Gliederung der Leitlinie ist so aufgebaut, dass jeweils evidenz- oder konsensbasierte Aussagen zu spezifischen Zielsymptomen in Abhängigkeit von Alter und Intelligenzniveau gemacht werden. Für folgende Zielsymptome werden Aussagen getroffen:

a. Soziale Interaktion und Kommunikation

b. Repetitives Verhalten, Sonderinteressen und sensorische Hyper-/Hyporeaktivität

c. Kognitive und alltagspraktische Fertigkeiten sowie adaptives Verhalten

d. Spezifische komorbide Entwicklungsstörungen (Sprach- und Sprechstörungen, umschriebene Entwicklungsstörungen der motorischen Funktionen, Ausscheidungsstörungen)

e. komorbide psychische Störungen und Symptome (oppositionelles und aggressives Verhalten/Störungen des Sozialverhaltens; Aktivitäts- und Aufmerksamkeitsstörung /hyperaktives Verhalten (ADHS); Angststörungen; depressive Episode, rezidivierende depressive Störung; Zwangsstörungen; Tic-Störungen; Schlafstörungen; Essstörungen; psychotische Störungen; bipolare Störungen; Persönlichkeitsstörungen; Suchterkrankungen; abnorme Gewohnheiten und Störungen der Impulskontrolle, insbesondere pathologisches Spielen und pathologischer Medienkonsum).

Die empfohlenen Interventionen für die unterschiedlichen Therapieziele können im Rahmen der konkreten Therapie kombiniert werden. Es muss individuell, basierend auf der konkreten diagnostischen Abklärung, entschieden werden, ob Therapieziele und die zugehörigen 
Methoden parallel eingesetzt werden können oder ob ein hierarchisches Vorgehen gewählt werden muss.

4. Festlegung des Behandlungssettings: Im Rahmen des Fallmanagements soll festgelegt werden, ob die aktuell notwendige Therapie im ambulanten, teil- oder vollstationären Rahmen stattfinden sollte bzw. ob Interventionen im sonderpädagogischen Setting oder im Rahmen eines strukturierten Beschäftigungsangebotes durchgeführt werden sollten.

5. Weitere Unterstützungsmöglichkeiten: Im Rahmen des Fallmanagements soll außerdem regelmäßig festgelegt und überprüft werden, welche weiteren Unterstützungsmöglichkeiten eingeführt bzw. fortgesetzt oder beendet werden sollen (Einbezug bzw. Unterstützung von Familien und Angehörigen; Unterstützung in Kindergarten und Schule, Arbeit, bzgl. der Wohnsituation/des Wohnens, bzgl. des Freizeitverhaltens).

6. Verlaufskontrollen: Es sollen regelmäßige Verlaufskontrollen einschließlich der entsprechenden diagnostischen Abklärung bezüglich des Erreichens der konkreten Therapieziele durchgeführt werden. Diese Verlaufskontrollen sollten - je nach Therapieziel und Intervention - zwischen ca. 1/2 (Medikation) bis ca. 6 Monaten (psychosoziale Intervention) durchgeführt werden. Fortschritte sollten evaluiert werden, um den Erfolg der Therapie oder der unterstützenden Maßnahmen abschätzen zu können und um eine Entscheidung zu treffen, ob die Therapie/Maßnahmen weiter fortgeführt, intensiviert oder beendet werden sollten oder ob andere Interventionen/Maßnahmen sinnvoll sind. Falls im Rahmen der Verlaufskontrolle oder über die Lebensspanne zusätzliche Symptome oder Diagnosen entstehen (was die Regel ist im Lebensverlauf von Personen mit Autismus-Spektrum-Störung), soll eine erneute Evaluation einschließlich entsprechender Diagnostik, Indikationsstellung und Therapieplanung sowie befristeter Intervention bezüglich der konkreten Therapieziele zu diesem Zeitpunkt durchgeführt werden.

Dieses Vorgehen impliziert ein sehr strukturiertes, kleinschrittiges und zielorientiertes Vorgehen, das von der aktuellen klinischen Praxis erheblich abweicht, jedoch das große Potenzial besitzt, die Versorgung von Menschen mit Autismus-Spektrum-Störungen deutlich zu verbessern.

- Veränderungen sind nicht frei von Emotionen, sie lösen häufig Verunsicherung, Angst, Ärger oder Widerstand aus, insbesondere bei denen, die sie betreffen und die die Veränderung nicht selbst initiiert haben. Hat bei der ablehnenden Haltung gegenüber der Autismus-Thera- pieleitlinie ein Sicherungsmechanismus eingesetzt, der in defensiver Weise den gegenwärtigen Status verteidigt und das Potenzial und die positiven Aspekte der Leitlinie außer Acht lässt?

- Entscheidend für die Annahme von Veränderungen ist, wie diese kommuniziert werden. Ist diese Kommunikation bereits vor der Veröffentlichung gescheitert?

Aufseiten der Anbieter bzw. Leistungserbringer von Autismus-Therapien besteht die Sorge, dass die Publikation der S3-Leitlinie den Zugang zur individuellen Versorgung Betroffener erschweren könnte, da Therapien dann z.B. nicht mehr in der bisher üblichen Zeitdauer, dem bisherigen Setting und häufig ohne externe Verlaufskontrollen durchgeführt werden könnten. Zum Beispiel empfiehlt die Leitlinie bei älteren Kindern und Jugendlichen mit Intelligenzminderung nach dem Grundschulalter zur Förderung der sozialen Interaktion und Kommunikation eine zeitlich begrenzte Gruppentherapie über 3 bis 6 Monate unter Einbezug der Eltern sowie der Schule. Da es zur Wirksamkeit dieser Intervention jedoch nur wenige Studien gibt, wird dies als „Kann“-Empfehlung formuliert. Zur Wirksamkeit von Einzeltherapien zur Förderung der sozialen Interaktion mit Gleichaltrigen liegen dagegen bis dato keinerlei evidenzbasierte Wirksamkeitsnachweise vor, sodass diese Therapieform nicht empfohlen wird. Dieser Empfehlung schloss sich die Leitlinienkommission mit lediglich einer Gegenstimme an, sie steht jedoch in deutlichem Kontrast zur üblichen Praxis, bei der sehr viele Betroffene mit/ ohne Intelligenzminderung über einen langen Zeitraum Einzeltherapie erhalten und dabei meist sehr allgemeine, allumfassende und "ganzheitliche" Therapieziele genannt werden (z.B. Entwickeln von Kontakt- und Beziehungsfähigkeiten, Kommunikation und Selbstbestimmung). In einem entsprechenden „Positionspapier" macht der Bundesverband ,autismus Deutschland e.V.“" klar, dass es sich bei der ambulanten therapeutischen Förderung nicht um ein ,isoliertes Funktionstrainings, sondern um komplexe Maßnahmen zur Eingliederung und Teilhabe" handle. Es seien ,ausreichende zeitliche Ressourcen erforderlich: Die Maßnahmen müssen zum einen langfristig angelegt sein (i.d.R. über mehrere Jahre). Zum anderen sollte ein zeitlicher Rahmen zur Verfügung stehen, der ein regelmäßiges und intensives Arbeiten ermöglicht. Maßnahmen, die den hier skizzierten quantitativen Mindeststandard unterschreiten, sind im Hinblick auf das Ziel der Verbesserung und Sicherstellung der Teilhabe der im Fokus stehenden Menschen mit Autismus-Spektrum-Störung in der Regel nicht zielführend.“8 Diese Aussagen spiegeln zwar die sub- 
jektive, therapeutische Erfahrung von in der Versorgung Tätiger wider - es liegen jedoch bezüglich „ganzheitlicher Therapieziele“, „komplexer" Maßnahmen“ und dem notwendigen „langen zeitlichen Rahmen“ keinerlei empirisch abgesicherte und qualitativ hochwertige Daten vor, die diese Aussagen stützen könnten.

Beim Prozess der Erstellung wissenschaftlicher Leitlinien dürfen versorgungspolitische Erwägungen die empirisch geleiteten Empfehlungen nicht verzerren. Dies würde die Aussagen der Leitlinien aus dem wissenschaftlichen in den politischen Bereich verschieben und damit die Evidenzbasierung der Empfehlungen ad absurdum führen. Das gilt insbesondere für die methodisch anspruchsvollen und auf höchstem Niveau angesiedelten S3-Leitlinien (evidenz- und konsensbasiert; repräsentativ, strukturiert und systematisch).

-Was würde ein im Vorhinein abgegebenes Votum gegen die Leitlinie über diejenigen aussagen, die das Votum aussprechen? Bedeutet dies eine Negation empirischer Evidenz?

\section{Ausblick}

Die „Qualität“ einer Leitlinie hängt erheblich von den methodischen Ansprüchen ab, aber auch von der professionellen Akzeptanz der Leitlinie. Es besteht daher die berechtigte Sorge, dass die Leitlinie nur mäßig Anwendung findet, wie dies auch bei anderen Leitlinien der Fall ist (siehe z.B. Ergebnisse zur AWMF-Leitlinie zu Hyperkinetischen Störungen [Isensee, Hagmayer, Rothenberger, Rothenberger \& Becker, 2015] oder der klinischen Leitlinie zur Empfehlung des Vorrangs der psychotherapeutischen Behandlung von ADHS im Vorschulalter vor der Behandlung mit Psychopharmaka [Chung, Tchaconas, Meryash \& Adesman, 2016]). Das grundsätzliche Problem, ob und inwieweit eine Leitlinie in der täglichen Routine ankommt, d.h. akzeptiert und umgesetzt wird, ist komplex (Lehmkuhl \& Holtmann, 2014) und dieser Prozess beginnt in Bezug auf die Autismus-Therapieleitlinie jetzt. Die Akzeptanz hängt einerseits von der Kommunikation (s.o.) ab, mit der die Leitlinie präsentiert und deren Empfehlungen und empirische Basis nachvollziehbar erklärt werden. Diese Kommunikation sollte wertschätzend sein und die gegenwärtige Versorgungssituation und ihre Vertreterinnen und Vertreter angemessen adressieren. Andererseits hängt die Akzeptanz aber auch von der Bereitschaft ab, die gegenwärtige Versorgungssituation gemeinsam verbessern und nicht einen Status quo erhalten zu wollen. Entscheidend ist auch die Verankerung und Vermittlung eines leitlinienorientierten Vorgehens in der klinischen Ausund Weiterbildung, um diese in der Versorgungsroutine $\mathrm{zu}$ etablieren (Lehmkuhl \& Holtmann, 2014). Notwendig sind weitere geeignete Implementierungsstrategien, wie z.B. eine Kurzversion, Algorithmen und Entscheidungsbäume usw., um das zu erreichen, was eigentlich alle wollen: eine bessere Versorgung der von Autismus Betroffenen. Der britische Kinder- und Jugendpsychiater Argyris Stringaris schrieb 2013 in einem Editorial zum ebenfalls bereits vor Veröffentlichung heftig befehdeten DSM-5: „The new DSM is coming - it needs tough love ..... Der Ausdruck ist im Deutschen wie so oft nicht ohne Weiteres übersetzbar. Was er aber meinte, war: Man kann und soll eine von vielen Fachleuten und Betroffenen in einem jahrelangen und hoch professionellen Prozess erarbeitete Richtlinie natürlich kritisieren; man kann für die Zukunft Veränderungsvorschläge machen; man kann weitere Forschung vorantreiben, um die Evidenzlücken zu füllen und die Evidenzlage zu verbessern. Aber man kommt um eine - noch so kritische - Annahme solcher Richtlinien nicht herum. Weil wir sie brauchen, um unsere Patientinnen und Patienten gut zu versorgen. Wir sind davon überzeugt, dass die S3-Leitlinie zur Therapie der AutismusSpektrum-Störungen dieses Potenzial hat.

\section{Literatur}

Arbeitsgemeinschaft der Wissenschaftlichen Medizinischen Fachgesellschaften (AWMF) - Ständige Kommission Leitlinien. (2016). Autismus-Spektrum-Störungen im Kindes-, Jugendund Erwachsenenalter - Teil 1: Diagnostik. Interdisziplinäre S3Leitlinie der DGKJP und der DGPPN sowie der beteiligten Fachgesellschaften, Berufsverbände und Patientenorganisationen. Verfügbar unter https://www.awmf.org/uploads/tx_szleitlini en/028-018l_S3_Autismus-Spektrum-Stoerungen_ASS-Diag nostik_2016-05.pdf

Bauer, M., Monz, B.U., Montejo, A. L., Quail, D., Dantchev, N., Demyttenaere, K. et al. (2008). Prescribing patterns of antidepressants in Europe: Results from the Factors Influencing Depression Endpoints Research (FINDER) study. European Psychiatry, 23, 66-73

Chung, J., Tchaconas, A., Meryash, D. \& Adesman, A. (2016). Treatment of attention-deficit/hyperactivity disorder in preschoolage children: Child and adolescent psychiatrists' adherence to clinical practice guidelines. Journal of Child and Adolescent Psychopharmacology, 26, 335-343.

Freitag, C.M, Jensen, K., Teufel, K., Luh, M., Todorova, A., Lalk, C. et al. (2020). Empirisch untersuchte entwicklungsorientierte und verhaltenstherapeutisch basierte Therapieprogramme zur Verbesserung der Kernsymptome und der Sprachentwicklung bei Klein- und Vorschulkindern mit Autismus-Spektrum-Störungen. Zeitschrift für Kinder- und Jugendpsychiatrie und Psychotherapie, 48, 224-243.

Höfer, J., Bachmann, C., Kamp-Becker, I., Poustka, L., Roessner, V., Stroth, S. et al. (2019). Willingness to try and lifetime use of complementary and alternative medicine in children and adolescents with autism spectrum disorder in Germany: A survey of parents. Autism, 23, 1865-1870.

Höfer, J., Hoffmann, F. \& Bachmann, C. (2016). Use of complementary and alternative medicine in children and adolescents with 
autism spectrum disorder: A systematic review. Autism, 21 , 387-402.

Hübner, L. \& Wienke, A. (2019). Fehlende Justiziabilität medizinisch-wissenschaftlicher Leitlinien. Eine Entscheidung des Oberlandesgerichts. DGNeurologie, 5, 422-424.

Isensee, C., Hagmayer, Y., Rothenberger, A., Rothenberger, L.G. \& Becker, A. (2015). AWMF-Leitlinie zu Hyperkinetischen Störungen in der Praxis. Zeitschrift für Kinder- und Jugendpsychiatrie und Psychotherapie, 43, 91-100.

Kamp-Becker, I., Stroth, S., Stehr, T. \& Weber, L. (2020). Segen oder Fluch? Das Internet als Informationsquelle über Autismus und Asperger-Syndrom. Zeitschrift für Kinder- und Jugendpsychiatrie und Psychotherapie, 48, 133-143.

Kopp, I.B., Selbmann, H.K. \& Koller, M. (2007). Konsensusfindung in evidenzbasierten Leitlinien - vom Mythos zur rationalen Strategie. Zeitschrift für Ärztliche Fortbildung und Qualitätssicherung, 101, 89-95.

Lehmkuhl, G. \& Holtmann, M. (2014). Umfassend, handhabbar, finanzierbar? Chancen und Herausforderungen bei der Erstellung, Akzeptanz und Anwendung von Leitlinien. Zeitschrift für Kinderund Jugendpsychiatrie und Psychotherapie, 42, 385-388.

Poustka, L. \& Kamp-Becker, I. (2017). Current practice and future avenues in autism therapy. Current Topics in Behavioral Neurosciences, 30, 357-378.

Poustka, L., Poustka, F. \& Kamp-Becker, I. (2019). AutismusSpektrum-Störungen. In S. Schneider \& J. Margraf (Hrsg.), Lehrbuch der Verhaltenstherapie, Band 3: Psychologische Therapie bei Indikationen im Kindes- und Jugendalter. Berlin: Springer.

Schepker, R. (2018). Von Sinn und Grenzen von Leitlinien - Werkstattbetrachtungen. Zeitschrift für Kinder- und Jugendpsychiatrie und Psychotherapie, 46, 264-265.

Stringaris, A. (2013). The new DSM is coming - it needs tough love ... Journal of Child Psychology and Psychiatry, 54, 501-502.

Teufel, K., Wilker, C., Valerian, J. \& Freitag, C.M. (Hrsg.). (2017). AFFIP - Autismusspezifische Therapie im Vorschulalter. Berlin: Springer.

\section{Prof. Dr. Inge Kamp-Becker}

Klinik für Kinder- und Jugendpsychiatrie, Psychosomatik und

Psychotherapie

Fachbereich Medizin der Philipps-Universität Marburg

Hans-Sachs-Str. 4

35039 Marburg

Deutschland

kampbeck@med.uni-marburg.de 